Reprod. Nutr. Develop., 1988, 28 (3 A), 603-615

\title{
Evolution au cours du temps de la composition en chaînes alkényles et acyles des plasmalogènes de mitochondries de cœur et de reins chez des rats ingérant de la triélaïdine
}

\author{
R. L. WOLFF (1), Nicole A. COMBE, B. ENTRESSANGLES
}

Unité de Biochimie-Biotechnologie ITERG, Laboratoire de Lipochimie Alimentaire, Département Alimentation et Nutrition, Université de Bordeaux I Avenue des Facultés, 33405 Talence Cedex, France.

Summary. Time-course changes in the alkenyl and acy/ chain profile of plasmalogens from heart and kidney mitochondria of rats fed elaidic acid.

Weaned rats were fed for 7 or 32 days a semi-purified diet containing $10 \%$ of fat supplement in which elaidic acid accounted for $64.5 \%$ of total fatty acids. Alkenyl groups from plasmalogens of kidney and heart mitochondria were analysed as their alkylsubstituted dioxane derivatives by gas liquid chromatography (GLC). Acyl chains of total and individual phospholipids (phosphatidylcholine: PC; phosphatidylethanolamine: PE, diacyl and alkenyl-acyl forms; phosphatidylinositol: $\mathrm{Pl}$; cardiolipin: $\mathrm{CL}$ ) were also analysed by GLC. Maximum level of elaidic acid incorporation was attained after only one week on the experimental diet in either total or individual phospholipids. Heart mitochondria phospholipids contained about $40 \%$ more trans-monounsaturated acids $(20.9 \%)$ than kidney mitochondria phospholipids $(12.5 \%)$ after one month. trans-Monounsaturated acids ( $t-18: 1$ p/us $t-16: 1$ acids) were incorporated in individual phospholipids from both organs in decreasing order: $\mathrm{PI}>\mathrm{PE}$ (total) $>\mathrm{PC} \gg \mathrm{CL}$. The order of incorporation of elaidic acid seemed to be related to the content of stearic acid in these phospholipids when the diet was devoid of elaidic acid ( $\mathrm{PI}>\mathrm{PE}>\mathrm{PC} \gg \mathrm{CL}$ ). The content of elaidic acid in alkenyl-acyl-glycerophosphoethanolamine (alkenyl-acyl-GPE) was the same ( $6 \%$ of total fatty acids) in both organs.

The time-course incorporation of trans-monounsaturated alkenyl chains was different. After one week on the elaidic acid-enriched diet, they represented only $65 \%$ (kidney) or $43 \%$ (heart) of the values reached after one month. At the end of the feeding period, the trans-monounsaturated alkenyl chains were also about $40 \%$ less in total plasmalogens from kidney mitochondria $(27,8 \%)$ than from heart mitochondria $(46 \%)$. trans-Monounsaturated acyl chains were incorporated at a higher rate in the 2-position of alkenyl-acylGPE than trans-monounsaturated alkenyl chains in their 1 -position. Our results confirm the unique observation of Kramer et al. (1985) who noticed that the fatty acid composition of phospholipids was much more rapidly equilibrated than the alkenyl chain composition of

( ${ }^{1}$ ) A qui envoyer la correspondance. 
plasmalogens (1 week vs 2-3 weeks) from heart of rats fed diets supplemented with different oils.

\section{Introduction.}

La majorité des acides gras insaturés apportés par l'alimentation est de configuration cis, mais dans le cas des matières grasses laitières, et surtout dans celui des huiles partiellement hydrogénées, une proportion parfois importante de ces acides peut être de configuration trans (Entressangles, 1986). On sait, depuis plus de cinquante ans (Sinclair, 1935), que ces acides sont utilisés par l'organisme et incorporés dans les phospholipides de la plupart des organes (Wood, 1979) et dans ceux de diverses fractions subcellulaires (Decker et Mertz, 1966 ; Sgoutas et al., 1973; Hsu et Kummerow, 1979; Høy et Hølmer, 1979 ; Blomstrand et Svensson, 1983; Wolff et al., 1984).

Il existe cependant une catégorie de phospholipides qui ne semble pas avoir, dans de telles études, attiré l'attention des chercheurs. Ce sont les plasmalogènes, ou alkényle-acyle-glycérophospholipides. Ces constituants membranaires, dont on a pu calculer qu'ils constituent près de $19 \%$ de l'ensemble des phospholipides du corps humain (Horrocks et Sharma, 1982), se distinguent des phospholipides diacylés plus communs tels que la diacyle-glycérophosphocholine (diacyle-GPC) ou la diacyle-glycérophosphoéthanolamine (diacyle-GPE) par la présence, en position 1 du $s n$-glycérol, de chaînes aliphatiques engagées dans une liaison vinyl-éther. Ces chaînes alkényles dérivent d'alcools gras, qui proviennent eux-mêmes d'acides gras après réduction par l'acyle-CoA-réductase (Horrocks et Sharma, 1982). Par conséquent, on peut prévoir que la composition des chaînes alkényles dépend, dans une certaine mesure, des acides gras alimentaires. De fait, quelques rares observations ont montré qu'il en était bien ainsi (Kramer et Hulan, 1977 ; Mukherjee et Kiewitt, 1980 ; Wolff et al., 1985, 1987 ; Kramer et al., 1985). En ce qui concerne les acides gras trans-mono-insaturés d'origine alimentaire, il a été clairement démontré qu'ils pouvaient s'incorporer dans les plasmalogènes du cœur entier (Mukherjee et Kiewitt, 1980), et, au niveau subcellulaire, dans les plasmalogènes des mitochondries de cœur (Wolff et al., 1985) et de reins (Wolff et al., 1987). On a également observé, dans des cellules LM en culture, que la supplémentation en acide élaïdique du milieu provoquait une accumulation d'alcool élaïdique (Lee et al., 1979 ; Lee et Stephens, 1982) et que l'addition d'un mélange de trans-octadécénols au milieu de culture de cellules ascitiques $L 1210$ et $S 180$ (ou leur injection dans le cerveau du rat) se traduisait par l'apparition de ces composés dans les plasmalogènes (Weber et Richter, 1982).

Dans des travaux récents (Wolff et al., 1985, 1987), il a été montré que l'effet majeur de l'ingestion d'acide élaïdique sur les chaînes alkényles des plasmalogènes de mitochondries de cœur ou de reins de rats était une substitution des chaînes alkényles saturées par des chaînes alkényles dérivées de l'acide élaïdique ou de son produit de raccourcissement, l'acide palmitélaidique. Cet effet était similaire à celui observé sur les chaînes acyles de la phosphatidylcholine (PC) ou 
de la phosphatidyléthanolamine (PE) des mitochondries de cœur ou de reins, dans lesquelles l'acide élaïdique se substituait aux acides palmitique et stéarique (Wolff et al., 1984, 1987).

Par le jeu des enzymes de désacylation-réacylation, l'incorporation de l'acide élaïdique dans les phospholipides diacylés est rapide: un taux maximum est atteint dès la première semaine chez des rats ingérant de la triélaïdine, taux qui est maintenu jusqu'à un mois (Wolff et al., 1984). Mais dans le cas particulier des plasmalogènes, il ne peut y avoir d'échange des chaînes alkényles; I'alcool gras est incorporé dès la première étape du métabolisme de novo de ces composés (Horrocks et Sharma, 1982), lors de l'alkylation de l'acyle-dihydroxyacétonephosphate (acyle-DHAP). Par conséquent, il nous a paru intéressant de suivre, au cours du temps, l'apparition des chaînes alkényles trans-mono-insaturées dans les plasmalogènes de mitochondries de cœur et de reins de rats, et de comparer cette incorporation à celle de l'acide élaïdique dans les phospholipides totaux ou individuels de ces organites, ainsi que dans les chaînes acyles des plasmalogènes à éthanolamine.

\section{Matériel et méthodes.}

Animaux et régimes. - Vingt rats mâles de souche Wistar, pris au sevrage, ont été nourris avec un régime composé de $90 \%$ d'un aliment purifié alipidique (UAR, Villemoisson-sur-Orge, France) et de $10 \%$ en poids d'un mélange de trioléine élaïdisée, d'huiles de tournesol et de lin, dont la composition en acides gras était la suivante : $16: 0: 1,1 \% ; 18: 0: 0,6 \% ; 18: 19$-trans (acide élaïdique) : $64,5 \% ; 18: 1$ cis : $24,9 \% ; 18: 2(n-6): 7,1 \% ; 18: 3(n-3): 0,7 \%$. La composition détaillée de l'aliment commercial de base a été décrite précédemment(Wolff et al., 1984). La moitié des animaux a reçu ce régime pendant une semaine, et l'autre moitié pendant 32 jours.

Préparation des mitochondries et extraction des lipides. - A l'issue des périodes expérimentales, les animaux ont été décapités après anesthésie légère par l'oxyde d'éthyle et leurs cœur et reins immédiatement prélevés. Les mitochondries de cœur et de reins ont été préparées par centrifugation différentielle, à partir des organes réunis et homogénéisés, selon des méthodes décrites précédemment (Wolff et al., 1984, 1985). La pureté des mitochondries de cœur, déterminée par les marqueurs enzymatiques habituels (Comte et Gautheron, 1978) et par microscopie électronique, est supérieure à $90 \%$, et celle des mitochondries rénales, estimée par la teneur en cardiolipides (Zambrano et al., 1975), est de $70 \%$ environ. Les lipides ont été extraits dès l'obtention des culots de mitochondries selon une adaptation (Wolff et al., 1987) de la méthode de Folch et al. (1957) et conservés en présence de 2,6-di-tert-butyl-4-méthyl-phénol (BHT) à $-20^{\circ} \mathrm{C}$ jusqu'à leur utilisation.

Analyse des chaines alkényles des plasmalogènes totaux. - Les phospholipides totaux ont été séparés des lipides apolaires par chromatographie sur couche mince ( $\mathrm{CCM}$ ) de silicagel (Kieselgel H 60, Merck, Dartmstadt, R.F.A.) à l'aide du 
mélange oxyde d'éthyle/acétone $(60: 20, \mathrm{~V} / \mathrm{V})$. La zone de gel correspondant aux phospholipides, qui demeurent sur la ligne de dépôt, a été grattée et les phospholipides ont été extraits du gel à l'aide successivement de chloroforme/méthanol $(2: 1, V / V)$, de méthanol, et de méthanol/eau $(9: 1, V / V)$ (Wolff et al., 1985).

Les chaînes alkényles des plasmalogènes totaux ont été converties en dérivés triméthylène-dioxy-alkanyles (TMDOA) par action du propane-diol-1,3 sur les phospholipides totaux en présence d'acide toluènesulfonique- 4 dans du benzène sec, pendant $4 \mathrm{~h}$ à $80^{\circ} \mathrm{C}$ et sous agitation (Palmer et al., 1981). Après extraction du milieu de réaction, les TMDOA ont été purifiés par CCM à l'aide du mélange hexane/oxyde d'éthyle/acide acétique (90:10:1, V/V/V) et extraits du silicagel par de l'hexane, après avoir mis le gel en suspension dans $1 \mathrm{ml}$ de mélange méthanol/eau/acide acétique $(10: 10: 1, \mathrm{~V} / \mathrm{V} / \mathrm{V})$ (Wolff et a/., 1985).

Analyse des chaînes acyles des phospholipides. - Les phospholipides totaux ont été séparés des lipides apolaires comme indiqué précédemment et leurs acides gras transméthylés directement en présence de gel (voir infra).

Les phospholipides individuels ont été séparés par CCM de silicagel dans le système de solvants chloroforme/méthanol/eau (65:25:4, V/V/V) qui permet de séparer les cardiolipides $(C L)$, la $P E$, et le mélange $P C+$ phosphatidylinositol (PI). Les zones de gel contenant les $C L$ et la PE ont été grattées et utilisées telles quelles pour la transméthylation des acides gras. Le mélange $\mathrm{PC}+\mathrm{Pl}$ a été extrait du gel avec la même séquence de solvants que celle décrite plus haut pour les phospholipides totaux, et les deux phospholipides ont été séparés par CCM de silicagel à l'aide du mélange de solvants butanol-1/acide acétique/eau (60:20:20, $\mathrm{V} / \mathrm{V} / \mathrm{V}$ ).

Pour toutes les CCM de silicagel, les solvants utilisés renfermaient 0,02\% de BHT et la révélation des plaques était réalisée par vaporisation d'une solution de dichlorofluorescéine (DCF) dans l'éthanol à $95^{\circ}(0,2 \%, \mathrm{Pds} / \mathrm{V})$ et observation sous lumière UV $(254 \mathrm{~nm})$.

Pour l'analyse des chaînes acyles en position 2 du sn-glycérol des alkyleacyle-GPE et des chaînes acyles des diacyle-GPE, la PE (mélange de diacyle-GPE et d'alkényle-acyle-GPE) a été extraite du gel et la DCF éliminée (Wolff et al., 1985). Puis la PE a été dissoute dans $0,5 \mathrm{ml}$ d'oxyde d'éthyle et $0,3 \mathrm{ml}$ d'oxyde d'éthyle saturé en $\mathrm{HCl}$ ont été ajoutés pour rompre les liaisons vinyl-éther des plasmalogènes (Palmer et al., 1981). Après $30 \mathrm{~min}$ d'agitation à température ambiante, l'acide a été évaporé sous un courant d'azote (en évitant d'amener la solution à sec par addition permanente d'oxyde d'éthyle, et en vérifiant à l'aide d'un papier $\mathrm{pH}$ l'acidité des vapeurs) ainsi que l'oxyde d'éthyle. Les lysophospholipides issus des plasmalogènes correspondants et les diacyle-GPE ont été séparés par CCM dans le mélange de solvants chloroforme/méthanol/eau $(65: 25: 4, V / V / V)$.

Les acides gras des phospholipides (totaux ou individuels) ont été transformés en esters méthyliques (EM) suivant la méthode de Morrison et Smith (1964). 
Analyses des TMDOA et des EM par chromatographie en phase gazeuse (CPG). - Les CPG des TMDOA et des EM ont été effectuées à l'aide d'appareils Varian 1400 et 940 munis de détecteurs à ionisation de flamme et équipés de colonnes d'acier inox ( $\varnothing$ ext. : 0,32 cm), remplies soit de gas-chrom Q (100-120 mesh) imprégné de $7 \%$ de butane-diol-succinate (BDS; longueur $3 \mathrm{~m}$ ), soit de chromosorb P-DMCS (80-100 mesh) imprégné de 15\% d'OV 275 (longueur : $6 \mathrm{~m}$ ). Les températures d'utilisation de ces colonnes étaient respectivement, de $185^{\circ} \mathrm{C}$ et $215^{\circ} \mathrm{C}$, et les gaz vecteurs, l'azote et l'hélium. La colonne d'OV 275 permet de séparer directement les TMDOA mono-insaturés cis de leurs isomères trans, sans fractionnement préalable sur plaque de silicagel imprégné de nitrate d'argent (Wolff et al., 1985, 1987).

Les identifications des TMDOA et des EM ont été faites selon un ensemble de méthodes décrites ailleurs (Wolff et al., 1985, 1987). Les valeurs présentées dans cette étude sont les moyennes des résultats d'analyses en CPG d'EM d'acides gras ou de TMDOA préparés à partir de deux échantillons de phospholipides (totaux ou individuels) isolés de façon indépendante à partir des lipides totaux de mitochondries des organes rassemblés de dix rats. Chaque préparation d'EM ou de TMDOA a été analysée une fois sur colonne de BDS et une fois sur colonne d'OV 275. Les proportions de chaînes mono-insaturées trans sont calculées à partir des pourcentages de chaînes monoénoïques établies sur colonne de BDS, en leur appliquant les proportions relatives des isomères cis et trans déterminées sur colonne d'OV 275. Cette façon d'opérer est justifiée par le fait que les colonnes d'OV 275, selon leur âge, ne permettent pas de quantifier correctement les acides gras poly-insaturés (Walker, 1981).

\section{Résultats et discussion.}

\section{Cinétique et effets de l'incorporation.}

Dans la figure 1 sont rassemblées, sous forme d'histogrammes, les compositions en chaînes alkényles des plasmalogènes totaux de mitochondries de reins et de cœur de rats ayant ingéré de l'acide élaidique pendant 7 ou 32 jours, ainsi que les compositions établies chez des animaux au sevrage ou ayant reçu un régime témoin (régime équilibré de laboratoire), dépourvu d'acides gras trans, pendant 6 à 8 semaines. Ces dernières données sont empruntées à des travaux récents (Wolff $e$ t al., 1985, 1987) et mentionnées à titre de comparaison. Pour les deux organes, chez les rats n'ayant pas ingéré d'acide élaidique, les compositions en chaînes alkényles sont relativement simples et limitées à des chaînes $16: 0$, $18: 0$ et $18: 1$ principalement. Pour ces dernières, deux isomères dérivés des acides oléique et cis-vaccénique sont présents; mais en raison de l'emploi de colonnes remplies pour les analyses en CPG, ils n'ont pu être séparés et quantifiés individuellement. En plus de ces composés, les plasmalogènes renferment des chaînes $18: 2$, et, en quantités mineures $(<1 \%)$, des chaînes insaturées à 15 et 17 atomes de carbone (non mentionnées dans la figure 1). Qualitativement, ce profil simple des chaînes alkényles est comparable au profil des chaînes acyles 
estérifiées en position 1 du $s n$-glycérol des diacyle-glycérophospholipides, composées d'acides $16: 0,18: 0$ et $18: 1$ également. On peut noter que les compositions en chaînes alkényles des plasmalogènes totaux des mitochondries de reins ou de cœur sont très voisines chez les animaux n'ayant pas consommé d'acides gras trans (fig. 1) : les chaînes $16: 0$ représentent environ la moitié de la totalité des chaînes alkényles, et les chaînes 18 : 0 et $18: 1$ environ 20 \% chacune, dans les deux organes.

Chez des rats ayant ingéré de l'acide élaïdique, on constate une incorporation progressive des chaînes alkényles trans-mono-insaturées, accompagnée d'une diminution presque équivalente des chaînes alkényles saturées. Après un mois de régime, les plasmalogènes de reins renferment $22,6 \%$ et $5,2 \%$ de chaînes alkényles dérivées, respectivement, de l'acide élaïdique et de son produit de raccourcissement, l'acide palmitélaïdique. Les valeurs correspondantes, dans le cas du cœur, sont de $44,5 \%$ et $1,5 \%$. Les valeurs dans le cas du rein sont identiques à celles que nous avions déterminées précédemment (Wolff et al., 1987) chez des animaux nourris pendant un ou deux mois avec un régime renfermant $10 \%$ d'un corps gras dont la teneur en acide élaïdique était de 69,0\%, et dans le cas du cœur, à celles déterminées après deux mois sur ce même régime (Wolff et al., 1985). En conséquence, il apparaît que la composition en chaînes alkényles des plasmalogènes est stabilisée dès le premier mois sur régime contenant de la triélaïdine.

En revanche, chez les animaux nourris pendant seulement une semaine avec le régime expérimental, les taux d'incorporation des chaînes alkényles trans-monoinsaturées sont moindres : dans les mitochondries de reins et de cœur, respectivement, $64 \%$ et $42 \%$ des valeurs atteintes au bout d'un mois. Cette situation est différente de celle présentée par les chaînes acyles des phospholipides (tabl. 1 et fig. 2). Dans les phospholipides totaux, en effet, un taux maximum d'incorporation des acides gras trans est atteint dès la première semaine et pratiquement maintenu jusqu'à un mois. Ce comportement peut être observé au niveau de chaque classe de phospholipides (tabl. 1) ; il est apparemment indépendant de la teneur finale en acide élaïdique qui est en revanche fonction de la nature de la tête polaire du phospholipide et de l'organe d'origine. Ainsi, l'incorporation d'acides gras trans est plus importante dans les phospholipides de mitochondries de cœur que dans ceux de reins, d'un facteur d'environ 1,5 à 2,2 à l'exception toutefois des alkényle-acyle-GPE et des CL où les taux d'incorporation sont identiques quel que soit l'organe d'origine. On notera que, pour les deux organes étudiés, l'acide élaïdique est mieux incorporé dans les diacyle-GPE ou les PI que dans les PC, et qu'il n'est que difficilement incorporé dans les $C L$. On peut également remarquer que l'acide palmitélaïdique n'est présent en quantités notables que dans les PC (et les CL dans le cas du rein) et pratiquement pas dans les PE et les PI.

Les plasmalogènes des mitochondries de cœur et de reins de rats sont essentiellement des plasmalogènes à éthanolamine (Gloster et Harris, 1970 ; Palmer et al., 1981 ; Wolff et al., 1984, 1985, 1987) et les compositions en TMDOA des plasmalogènes totaux décrites dans cette étude correspondent donc, globalement, à celles des plasmalogènes à éthanolamine. II apparaît que l'incorporation des chaînes $16: 1$ trans n'est pas liée à la nature de la tête polaire 

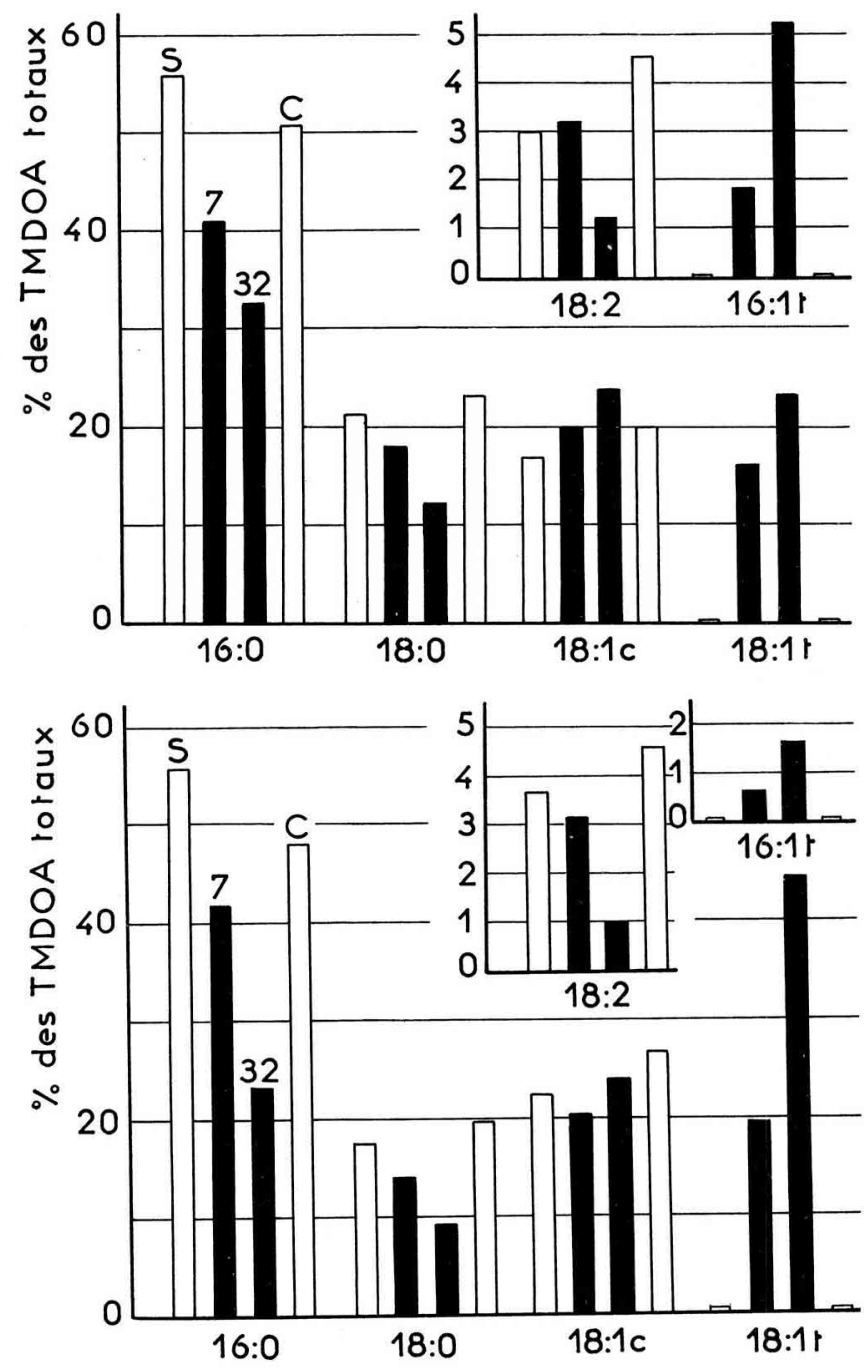

FIG. 1. - Compositions en chaînes alkényles (exprimées en \% des surfaces en CPG des TMDOA totaux) des plasmalogenes totaux de mitochondries de reins (en haut) et de coeur (en bas) de rats au sevrage (barres blanches: S), ayant reçu de l'acide élaïdique pendant 7 jours (barres noires: 7 ) ou 32 jours (barres noires: 32), ou ayant reçu un régime témoin pendant 6 à 8 semaines (barres blanches: C). Les valeurs pour les animaux au sevrage et pour les rats contrôles proviennent de publications antérieures (Wolff et al., 1985, 1987). Les valeurs sont les moyennes des résultats d'analyse en CPG des TMDOA préparés à partir de deux échantillons de phospholipides totaux isolés indépendamment à partir des lipides extraits des mitochondries des organes réunis de dix rats. Pour chaque préparation de TMDOA, une analyse a été effectuée sur colonne de BDS et une analyse sur colonne d'OV 275 . Les écarts à la moyenne sont au plus de $5 \%$ pour les TMDOA représentant $10 \%$ ou plus des TMDOA totaux, et de $10 \%$ au plus pour les TMDOA en représentant moins de $10 \%$. La structure des chaînes alkanyles des TMDOA (correspondant, à la double liaison en position 1 près, aux chaînes alkényles des plasmalogènes) est symbolisée par la formule $X$ : Yi où $X$ est le nombre de carbones, $Y$ le nombre de doubles liaisons, et i leur configuration $(\mathrm{c}=c i s, \mathrm{t}=$ trans $)$. 
TABLEAU 1

Taux d'incorporation des acides trans-mono-insaturés dans les phospholipides totaux et individue/s des mitochondries de reins et de cœur de rats ayant ingéré de l'acide élaïdique pendant des durées variables.

\begin{tabular}{|c|c|c|c|c|c|c|c|c|c|c|c|c|c|c|c|}
\hline \multirow{3}{*}{\multicolumn{2}{|c|}{ Organe $\mathrm{AG}^{\mathrm{a}}$}} & \multicolumn{14}{|c|}{ Nature du composé et durée du régime ${ }^{b}$} \\
\hline & & \multicolumn{2}{|c|}{ PL totaux } & \multirow{2}{*}{$\frac{P C}{7}$} & \multirow{2}{*}{\multicolumn{2}{|c|}{$\begin{array}{rl} & \text { PE : da } \\
327 & 7\end{array}$}} & \multirow{2}{*}{$\frac{+ \text { aa }}{32}$} & \multicolumn{2}{|c|}{ PE : da } & \multicolumn{2}{|c|}{ PE : aa } & \multirow{2}{*}{$\frac{\mathrm{PI}}{7}$} & \multicolumn{3}{|c|}{$\mathrm{CL}$} \\
\hline & & 7 & 32 & & & & & 7 & 32 & 7 & 32 & & 32 & 7 & 32 \\
\hline Rein & $\begin{array}{l}16 \\
18\end{array}$ & $\begin{array}{l}1 t 1,4^{\mathrm{c}} \\
1 t 12,4\end{array}$ & $\begin{array}{r}1,3 \\
11,2\end{array}$ & $\begin{array}{r}2,8 \\
13,7\end{array}$ & $\begin{array}{r}2,5 \\
11,3\end{array}$ & $\begin{array}{r}0,2 \\
15,0\end{array}$ & $\begin{array}{r}0,2 \\
14,3\end{array}$ & $\begin{array}{r}0,2 \\
16,7\end{array}$ & $\begin{array}{r}0,2 \\
14,7\end{array}$ & $\begin{array}{r}\mathrm{tr} \\
5,5\end{array}$ & $\begin{array}{r}\operatorname{tr} \\
6,1\end{array}$ & $\begin{array}{r}\text { tr } \\
18,8\end{array}$ & $\begin{array}{r}\text { tr } \\
16,0\end{array}$ & $\begin{array}{l}0,8 \\
0,6\end{array}$ & $\begin{array}{l}0,9 \\
0,7\end{array}$ \\
\hline Cœur & $\begin{array}{l}16 \\
18\end{array}$ & $\begin{array}{lll}1 & t & 0,4 \\
1 & t 20,9\end{array}$ & $\begin{array}{r}0,3 \\
20,6\end{array}$ & $\begin{array}{r}0,8 \\
23,5\end{array}$ & $\begin{array}{r}0,5 \\
21,7\end{array}$ & $\begin{array}{r}\text { tr } \\
27,9\end{array}$ & $\begin{array}{r}\text { tr } \\
27,0\end{array}$ & $\begin{array}{r}\mathrm{tr} \\
31,0\end{array}$ & $\begin{array}{r}\mathrm{tr} \\
32,7\end{array}$ & $\begin{array}{r}\mathrm{tr} \\
4,8\end{array}$ & $\begin{array}{r}\text { tr } \\
6,1\end{array}$ & $\begin{array}{r}\text { nd } \\
28,4\end{array}$ & $\begin{array}{r}\text { nd } \\
27,0\end{array}$ & $\begin{array}{r}\text { tr } \\
1,1\end{array}$ & $\begin{array}{r}\text { nd } \\
0,5\end{array}$ \\
\hline
\end{tabular}

a. Abréviations utilisées : $A G$ : acides gras; PL : phospholipides; PC, PE, PI, CL : voir texte ; da : forme diacyle; aa : forme alkényle-acyle; $\mathrm{tr}$ : traces $(<0,2 \%) ; 16: 1 t$ : acide palmitélaïdique, $18: 1 t$ : acide élaïdique; nd: composés non détectés. ${ }^{b}$. Durée, exprimée en jours. ${ }^{c}$. Les valeurs sont exprimées en \% en poids d'EM et représentent les moyennes des résultats d'analyse en CPG des EM d'acides gras correspondant à deux échantillons de chaque phospholipide. Chaque échantillon est isolé à partir des lipides totaux des mitochondries des organes réunis de 10 rats. Pour chaque échantillon de phospholipide, une analyse a été réalisée sur colonne de BDS et une analyse sur colonne d'OV 275 . Pour la clarté du tableau, les écarts à la moyenne $(<10 \%$ de la valeur indiquée pour l'ensemble des valeurs) ne sont pas mentionnés.
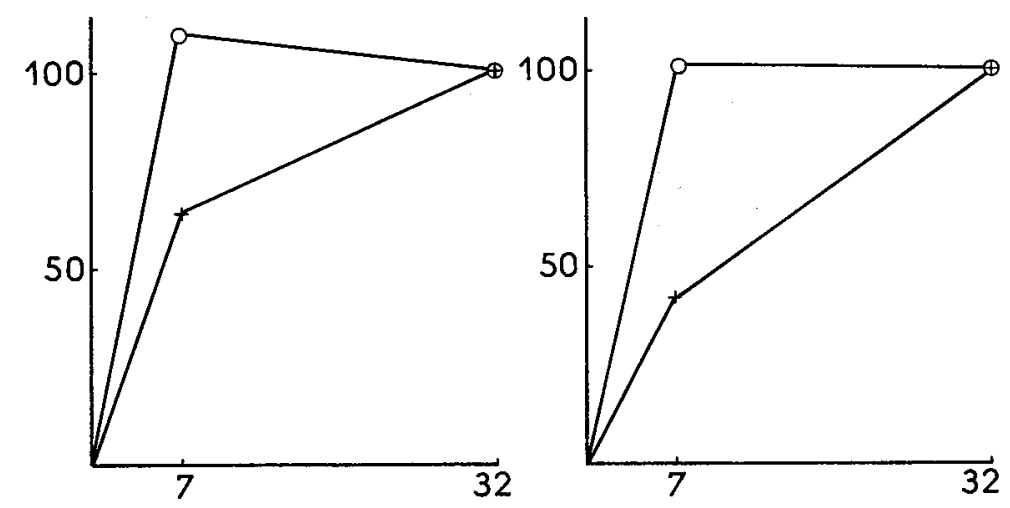

FIG. 2. - Comparaison des taux d'incorporation des chaînes trans-mono-insaturées totales dans les phospholipides totaux (chaînes acyles: $\bigcirc$ - - O) et dans les plasmalogènes totaux (chaines alkényles: + - + ), en fonction du temps (exprimé en jours) passé sur régime enrichi en acide élaidique: les taux sont exprimés en pourcentage de la valeur atteinte au bout d'un mois, prise égale à $100 \%$.

Figure de gauche: cas du rein; figure de droite : cas du cœur. 
du phospholipide, puisqu'on les retrouve préférentiellement dans les PC sous forme de chaînes acyles et dans les PE sous forme de chaînes alkényles. Il est plus probable, à notre sens, que ce qui oriente les chaînes 16:1 trans et $18: 1$ trans dans un phospholipide est la composition naturelle en acides gras saturés de ce phospholipide. Dans le cas du rein, par exemple, la PC des mitochondries renferme de 32 à $33 \%$ d'acide $16: 0$ et de 10 à $13 \%$ d'acide $18: 0$ (Wolff et al., 1987); ces acides gras étant principalement localisés en position 1 du snglycérol, ils représentent, par rapport aux acides gras estérifiés à cette position, de l'ordre de $60 \%$ pour l'acide $16: 0$ et de $25 \%$ pour l'acide $18: 0$. Ces proportions sont similaires à celles des chaînes alkényles en position 1 des plasmalogènes (fig. 1). Par contre, les acides palmitique et stéarique représentent de 9 à $13 \%$ et de 23 à $27 \%$, respectivement, des acides gras de la PE de mitochondries de rein (Wolff et al., 1987), soit environ $22 \%$ et $50 \%$ des acides gras estérifiés en position $1 \mathrm{du} s n$-glycérol, proportions inverses de celles relevées pour les chaînes alkényles des plasmalogènes. Ainsi, la $\mathrm{PC}$ et les plasmalogènes à éthanolamine ayant en position 1 des compositions en chaînes saturées similaires incorporent de la même façon les chaînes insaturées de configuration trans. Si l'on admet, dans le cas de la PC, que les acides gras trans sont incorporés en position 1 du sn-glycérol, ils représentent après un mois de régime, $5 \%$ pour l'acide palmitélaïdique, et $22,6 \%$ pour l'acide élaïdique, des acides gras estérifiés à cette position. Ces valeurs sont similaires à celles relevées pour les chaînes alkényles correspondantes dans les plasmalogènes.

Dans le cas du $\mathrm{PI}$, les acides $16: 0$ et $18: 0$ représentent normalement de 5 à $10 \%$ et de 40 à $50 \%$, respectivement, des acides gras totaux de ce phospholipide. Dans les $C L$, ces mêmes acides gras représentent, respectivement, de 3 à $5 \%$ et environ $1 \%$ de ses acides gras totaux. Les rapports $16: 0 / 18: 0$ sont inverses dans ces deux phospholipides, ce qui peut expliquer que l'acide $16: 1$ trans est bien incorporé dans les CL (relativement à l'acide $18: 1$ trans) alors qu'il ne l'est pas dans les PI.

Nos analyses nous ont également permis d'observer, pour la première fois à notre connaissance, que l'acide élaïdique est susceptible d'être estérifié en position 2 du sn-glycérol des plasmalogènes à éthanolamine. Cette position, chez des rats n'ayant pas ingéré d'acide élaïdique, est principalement occupée par des acides gras poly-insaturés (tabl. 2), surtout de la série (n-6) dans le cas du rein ( $80 \%$ ou plus) et des séries ( $n-6)$ ( $47 \%$ ou plus) et ( $n-3)$ (42\% environ) dans le cas du cœur. Pour ce dernier organe, les compositions que nous avons établies chez les rats élevés sur régime de laboratoire pendant un mois sont similaires à celles déterminées sur les plasmalogènes à éthanolamine préparés à partir de mitochondries de cœur de rat Sprague-Dawley adulte (Palmer et al., 1981). Toutefois, les taux d'incorporation de l'acide élaïdique demeurent faibles, même après un mois de régime, atteignant une valeur maximum de 6,1 \% dans les deux organes étudiés (tabl. 2). Cette relative difficulté d'estérification de la position 2 des plasmalogènes à éthanolamine par l'acide élaïdique est très probablement liée à la spécificité des transacylases qui contrôlent la composition des acides gras à cette position. Cela a d'ailleurs été démontré in vitro dans le cas de la diacyle-GPC, pour laquelle les vitesses d'acylation des positions 1 par les acides 
palmitique, stéarique, ou élaïdique, sont largement supérieures aux vitesses d'acylation des positions 2 par ces mêmes acides gras (Lands et al., 1966).

TABLEAU 2

Composition en acides gras (\% en poids) des alkény/e-acy/e-GPE de mitochondries de cœur et de reins de rats élevés sur régime contenant ou non de l'acide élaídique.

\begin{tabular}{|c|c|c|c|c|c|c|c|c|}
\hline & \multicolumn{4}{|c|}{ Coeur } & \multicolumn{4}{|c|}{ Rein } \\
\hline & $S^{a}$ & 7 & 32 & C & $S$ & 7 & 32 & C \\
\hline $16: 0^{b}$ & $3,1^{\mathrm{c}}$ & 2,5 & 2,8 & 1,7 & 4,6 & 4,1 & 4,3 & 3,9 \\
\hline $16: 1$ & 0,2 & 0,4 & 0,4 & $\mathrm{tr}^{\mathrm{d}}$ & 0,5 & 0,6 & 1,0 & 0,7 \\
\hline $18: 0$ & 4,5 & 2,2 & 2,5 & 3,0 & 5,6 & 6,0 & 4,1 & 7,1 \\
\hline $18: 1$ & 1,0 & 3,6 & 2,4 & 1,3 & 6,7 & 6,0 & 5,9 & 5,1 \\
\hline $18: 2(n-6)$ & 1,8 & 2,8 & 3,9 & 3,0 & 5,2 & 3,3 & 3,3 & 3,8 \\
\hline $20: 3(n-9)$ & nd & 0,1 & 1,3 & nd & $\operatorname{tr}$ & 0,2 & 1,6 & nd \\
\hline $20: 3(n-6)$ & 0,1 & 0,2 & 0,6 & $\operatorname{tr}$ & 1,2 & 0,4 & 0,8 & 0,5 \\
\hline $20: 4(n-6)$ & 41,2 & 38,3 & 56,0 & 45,7 & 64,9 & 65,3 & 67,3 & 72,5 \\
\hline $20: 5(n-3)$ & tr & 0,4 & 0,7 & 0,2 & 1,6 & 1,0 & 1,3 & 1,1 \\
\hline $22: 4(n-6)$ & 2,6 & 2,0 & 1,6 & 1,8 & 1,1 & tr & $\operatorname{tr}$ & 0,7 \\
\hline $22: 5(n-6)$ & 1,1 & 1,3 & 2,0 & 0,8 & $\operatorname{tr}$ & tr & tr & $\operatorname{tr}$ \\
\hline $22: 5(n-3)$ & 5,4 & 3,6 & 2,7 & 3,6 & $\operatorname{tr}$ & $\mathrm{tr}$ & $\operatorname{tr}$ & $\operatorname{tr}$ \\
\hline $22: 6(n-3)$ & 37,5 & 36,8 & 15,2 & 37,8 & 5,7 & 3,1 & 2,5 & 3,9 \\
\hline autres $^{\mathrm{e}}$ & 0,7 & 0,8 & 0,6 & 0,3 & 0,9 & 1,0 & 0,9 & 0,6 \\
\hline $16: 1 t$ & nd & 0,1 & 0,1 & nd & nd & $\operatorname{tr}$ & $\operatorname{tr}$ & nd \\
\hline $18: 1 t$ & nd & 4,8 & 6,1 & nd & nd & 5,5 & 6,1 & nd \\
\hline
\end{tabular}

. Désignations des régimes identiques à celles de la figure $1 .{ }^{b}$. Longueur, degré d'insaturation et série métabolique des acides gras. La configuration des doubles liaisons est cis, sauf indiqué ( $t$ : trans). ${ }^{c}$. Moyennes de 2 analyses, telles que définies dans les notes du tableau $1 .{ }^{d}$. Abréviations utilisées: $\operatorname{tr}$ : traces $(<0,1 \%)$; nd : composés non détectés. ${ }^{\mathrm{e}}$. Autres : sommes des acides $14: 0$, $15: 0,17: 0,20: 1$ et $20: 2(n-6)$, ne dépassant à aucun moment, pour chacun de ces acides, $0,4 \%$ des acides gras totaux.

\section{Implications métaboliques.}

La première étape de la biosynthèse des plasmalogènes consiste en une réaction d'alkylation de l'acyle-DHAP par un alcool gras, lui-même issu de la réduction d'un acide gras, activé sous forme d'acyle-CoA, par l'acyle-CoAréductase. Cette dernière enzyme présente une certaine spécificité vis-à-vis de la longueur et du degré d'insaturation des acides gras qui, couplée à la spécificité de l'alkyle-DHAP-synthase et à la nature des acides gras disponibles dans la cellule, conduit au profil simple des chaînes alkényles des plasmalogènes (Bishop et Hajra, 1978; Natarajan et Schmid, 1978).

La nature des acides gras alimentaires est un des facteurs majeurs responsables des variations de la composition en chaînes alkényles des plasmalogènes. Toutefois, cette influence n'a été que très peu étudiée. Kramer et al. (1985) ont montré que, selon la nature des corps gras ingérés, les chaînes alkényles des plasmalogènes à éthanolamine du cœur de rat pouvaient varier dans une très large 
mesure : de $27,8 \%$ à $56,1 \%$ pour les chaînes $16: 0$, de $22,5 \%$ à $41,2 \%$ pour les chaînes $18: 0$ et de 17,9 à $47,9 \%$ pour les isomères $(n-9)$ et $(n-7)$ des chaînes $18: 1$, c'est-à-dire pratiquement du simple au double pour chaque type de chaîne. Ces modifications sont stabilisées entre deux ou trois semaines après le sevrage chez des animaux élevés sur des régimes contenant divers corps gras (Kramer et al., 1985). Ce délai est du même ordre de grandeur que celui que nous avons établi dans cette étude (fig. 2) chez des rats ayant reçu de l'acide élaïdique. Cela indique que le temps nécessaire à l'équilibrage des compositions en chaînes alkényles n'est pas lié - au moins à l'échelle des temps considérés - à la configuration trans de la double liaison de l'acide élaïdique, mais au métabolisme propre des plasmalogènes. II n'existe pas en effet de mécanismes d'échange des radicaux alkényles, comme celui permettant le renouvellement des chaînes acyles des phospholipides, et les chaines alkényles présentes dans les plasmalogènes correspondent à des chaînes incorporées lors de la synthèse de novo de ces composés. Par contre, nos résultats montrent que les chaînes acyles des plasmalogènes à éthanolamine ont leur propre vitesse de renouvellement, différente des chaînes alkényles: après une semaine de régime, les taux d'incorporation de l'acide $18: 1$ trans en position 2 représentent de 80 à $90 \%$ de ceux atteints après un mois (tabl. 2).

L'acide élaïdique, et de façon plus générale les acides gras trans-monoinsaturés, lorsqu'ils sont incorporés dans les phospholipides, peuvent en être éliminés si l'ingestion de ces composés cesse (Moore et al., 1980; Wolff et al., 1984). Toutefois, leur vitesse d'élimination est sensiblement plus lente que leur vitesse d'incorporation, phénomène que nous avions pu attribuer, en partie au moins, à l'accumulation des acides gras trans dans les triglycérides de réserve (Wolff et al., 1984). L'apparition des chaînes alkényles trans-mono-insaturées est près de deux fois moins rapide dans les plasmalogènes que l'apparition des chaînes acyles correspondantes dans les phospholipides diacylés. En conséquence, si le remplacement des chaînes saturées par des chaînes trans-monoinsaturées peut avoir des conséquences physiologiques sur les membranes cellulaires, on peut penser que ces effets seront plus lents à s'instaurer s'ils sont liés aux plasmalogènes que s'ils le sont aux phospholipides diacylés, mais aussi qu'ils persisteront plus longtemps après que l'ingestion des acides gras trans aura cessé.

Reçu en octobre 1987. Accepté en janvier 1988.

Remerciements. - Les auteurs remercient vivement Mme Isabelle Wolff-Augarde qui a bien voulu assurer, gracieusement, le traitement informatique du texte.

\section{Références}

BISHOP J. E., HAJRA A. K., 1978. Specificity of reduction of fatty acids to long chain alcohols by rat brain microsomes. J. Neurochem., 30, 643-647. 
BLOMSTRAND R., SVENSSON L., 1983. The effects of partially hydrogenated marine oils on the mitochondrial function and membrane phospholipid fatty acids in rat heart. Lipids, 18 , $151-170$.

COMTE J., GAUTHERON D. C., 1978. The markers of pig heart mitochondrial sub-fractions: I. - The dual location of NADPH-cytochrome $c$ reductase in outer membrane and microsomes. Biochimie, 60, 1289-1298.

DECKER W. J., MERTZ W., 1966. Incorporation of dietary elaidic acid in tissues and effects on fatty acid distribution. J. Nutr. 89, 165-170.

ENTRESSANGLES B., 1986. Mise au point sur les isomères trans alimentaires. Rev. Fr. Corps Gras, 33, 47-58.

FOLCH J., LEES M., SLOANE-STANLEY G. H., 1957. A simple method for the isolation and purification of total lipides from animal tissues. J. biol. Chem., 226, 497-509.

GLOSTER J., HARRIS P., 1970. The lipid composition of mitochondrial and microsomal fractions of rat myocardial homogenates. Cardiovasc. Res., 4, 1-5.

HORROCKS L. A., SHARMA M., 1982. Plasmalogens and $O$-alkylglycerophospholipids, 51-93. In HAWTHORNE J. N. and ANSELL G. B., Phospholipids, Elsevier Biomedical Press.

HØY C. E., HØLMER G., 1979. Incorporation of cis and trans-octadecenoic acids into the membranes of rat liver mitochondria. Lipids, 14, 727-733.

HSU C. M. L., KUMMEROW F. A., 1977. Influence of elaidate and erucate on heart mitochondria. Lipids, 12, 486-494.

KRAMER J. K. G., FARNWORTH E. R., THOMPSON B. K., 1985. Cardiac lipid changes in rats fed oils enriched in saturates and their apparent relationship to focal heart lesions. Lipids, 20, 635-644.

KRAMER J. K. G., HULAN H. W., 1977. Changes in the acyl and alkenyl group composition of cardiac phospholipids in boars fed corn oil or rapeseed oil. Lipids, 12, 159-164.

LANDS W. E. M., BLANK M. L., NUTTER L. J., PRIVETT O. S., 1966. A comparison of acyltransferase activities in vitro with the distribution of fatty acids in lecithins in vivo. Lipids, 1, 224-229.

LEE T. C., HOUGLAND A. E., STEPHENS S., 1979. Perturbation of lipid metabolism in L-M cultured cells by elaidic acid supplementation : formation of fatty alcohols. Biochem. Biophys. Res. Com., 91. 1497-1503.

LEE T. C., STEPHENS S., 1982. The modification of lipid composition in L-M cultured cells supplemented with elaidate. Increased formation of fatty alcohols. Biochim. Biophys. Acta., 712, 299-304.

MOORE C. E., ALFIN-SLATER R. B., AFTERGOOD L., 1980. Incorporation and disappearance of trans fatty acids in rat tissues. Am. J. clin. Nutr., 33, 2318-2323.

MORRISON W. R., SMITH L. M., 1964. Preparation of fatty acid methyl esters and dimethylacetals from lipids with boron fluoride-methanol. J. Lipid Res., 5, 600-608.

MUKHERJEE K. D., KIEWITT I., 1980 . Incorporation of isomeric octadecenoic acids into alk-1-enyl moieties of cardiac glycerophospholipids of the rat. FEBS Lett., 122, 133-134.

NATARAJAN V., SCHMID H. H. O., 1978. Biosynthesis and utilization of long-chain alcohols in rat brain : aspects of chain-length specificity. Arch. Biochem. Biophys., 187, 215-222.

PALMER J.W., SCHMID P. C., PFEIFER D. R., SCHMID H. H. O., 1981. Lipids and lipolytic enzyme activities of rat heart mitochondria. Arch. Biochem. Biophys., 211, 674-682.

SGOUTAS D. S., JONES R., LEIGHT M., 1973. Distribution of dietary elaidic acid in subcellular particles of rat liver. Int. J. Biochem., 4, 437-445.

SINCLAIR R. G., 1935. The metabolism of the phospholipids. VIII. The passage of elaidic acid into tissue phospholipids. Evidence of the intermediary role of liver phospholipid in fat metabolism. J. biol. Chem., 111, 515-520.

WALKER B. L., 1981. The OV 275-packed stainless steel column in trans-fatty acid research : a note of caution. Lipids, 16, 468-471.

WEBER N., RICHTER I., 1982. Formation of ether lipids and wax esters in mammalian cells. Specificity of enzymes with regard to carbon chains of substrates. Biochim. Biophys. Acta, 711, 197-207.

WOLFF R. L., COMBE N., ENTRESSANGLES B., 1984. Incorporation et élimination de l'acide élaidique dans les différents phospholipides des mitochondries ou de leurs membranes chez le rat. Influence de l'organe et de l'âge. Rev. Fr. Corps Gras, 31, 161-170. 
WOLFF R. L., COMBE N., ENTRESSANGLES B., 1985. Modification of alkenyl chain profile in plasmalogens of rat heart mitochondria by dietary trielaidin. Lipids, 20, 367-372.

WOLFF R. L., COMBE N., ENTRESSANGLES B., MORIN O., 1987. Effets comparés de l'acide élaïdique alimentaire sur les chaînes alkényles et acyles des glycérophospholides de mitochondries de reins de rats. Rev. Fr. Corps Gras, 34, 525-532.

WOOD R., 1979. Distribution of dietary geometrical and positional isomers in brain, heart, kidney, liver, lung, muscle, spleen, adipose and hepatoma, 213-281. In EMKEN E. A. and DUTTON H. J., Geometrical and positional fatty acid isomers, The American Oil Chemists' Society, Champaign, llinois.

ZAMBRANO F., FLEISCHER S., FLEISCHER B., 1975. Lipid composition of the Golgi apparatus of rat kidney and liver in comparison with other subcellular organelles. Biochim. Biophys. Acta, 380. 357-369. 